\title{
Photodissociation Dynamics of Bromofluorobenzenes Using Velocity Imaging Technique
}

\author{
Ying Tang, ${ }^{\dagger}$ Wei-Bin Lee, ${ }^{\ddagger}$ Bing Zhang, ${ }^{\dagger}$ and King-Chuen Lin $*$ \\ State Key Laboratory of Magnetic Resonance and Atomic and Molecular Physics, Wuhan Institute of Physics \\ and Mathematics, Chinese Academy of Sciences, Wuhan 430071, P. R. China, and Department of Chemistry, \\ National Taiwan University, Taipei 106, and Institute of Atomic and Molecular Sciences, Academia Sinica, \\ Taipei 106, Taiwan
}

Received: October 22, 2007; In Final Form: December 4, 2007

\begin{abstract}
Velocity imaging technique combined with $(2+1)$ resonance-enhanced multiphoton ionization (REMPI) has been used to detect the $\mathrm{Br}$ fragment in photodissociation of $o-, m$-, and $p$-bromofluorobenzene at $266 \mathrm{~nm}$. The branching ratio of ground state $\operatorname{Br}\left({ }^{2} \mathrm{P}_{3 / 2}\right)$ is found to be larger than $96 \%$. Its translational energy distributions suggest that the $\mathrm{Br}$ fragments are generated via two dissociation channels for all the molecules. The fast route, which is missing in $p$-bromofluorobenzene detected previously by femtosecond laser spectroscopy, giving rise to an anisotropy parameter of $0.50-0.65$, is attributed to a direct dissociation from a repulsive triplet $\mathrm{T}_{1}\left(\mathrm{~A}^{\prime \prime}\right)$ or $\mathrm{T}_{1}\left(\mathrm{~B}_{1}\right)$ state. The slow one with anisotropy parameter close to zero is proposed to stem from excitation of the lowest excited singlet $\left(\pi, \pi^{*}\right)$ state followed by predissociation along a repulsive triplet $\left(\pi, \sigma^{*}\right)$ state localized on the $\mathrm{C}-\mathrm{Br}$ bond. For the minor product of spin-orbit excited state $\operatorname{Br}\left({ }^{2} \mathrm{P}_{1 / 2}\right)$, the dissociating features are similar to those found in $\operatorname{Br}\left({ }^{2} \mathrm{P}_{3 / 2}\right)$. Our kinetic and anisotropic features of decomposition obtained in $\mathrm{m}$ - and p-bromofluorobenzene are opposed to those by photofragment translational spectroscopy. Discrepancy between different methods is discussed in detail.
\end{abstract}

\section{Introduction}

Photochemistry of halogenated molecules including alkyl and aryl halides has received wide attention for decades, not only for their extreme importance to the environmental impact assessment but also for serving as a paradigm for research into fundamental photodissociation dynamics in the UV wavelength range. For alkyl halides, UV irradiation may lead to transition from a nonbonding electron of the halogen atom to the antibonding orbital of the $\mathrm{C}-\mathrm{X}$ bond ( $\mathrm{X}$ denotes halogen), thereby rapidly causing bond rupture. ${ }^{1-10}$ In contrast, the photodissociation processes for aryl halides are more complicated because more electronic states may be involved to make multiple dissociation channels probable. ${ }^{11-20}$ Their dissociation rates and channels rely on the type of halogen, substituents on the benzene ring and related positions, and excitation wavelengths.

Among the aryl halides, the simplest type of monohalobenzenes including chlorobenzene, ${ }^{21}$ bromobenzene, ${ }^{19,22}$ iodobenzene, ${ }^{11,12,15,23-25}$ and fluorobenzene ${ }^{26-28}$ have been widely studied. When the substituents on the benzene ring increase, their nature and positions may cause substantial impact on the photodissociation behavior. ${ }^{13,14,16,17,20,29-32}$ For instance, by using femtosecond pump-probe spectroscopy, Davidsson group investigated photodissociation dynamics of $o-, m-$, and $p$ dibromobenzene and 1,3,5-tribromobenzene at $266 \mathrm{~nm} .{ }^{18}$ The channel common for all these molecules was ascribed to predissociation via intersystem crossing (ISC). Its dissociation rate increases with the number of bromine atoms and with decreasing distance between the bromine atoms. The second dissociation channel, observed in $o$ - and $m$-dibromobenzene,

* Author to whom correspondence should be addressed. E-mail: kclin@ ccms.ntu.edu.tw. Fax: 886-2-23621483.

Chinese Academy of Sciences.

$\doteqdot$ National Taiwan University and Academia Sinica. was opened up due to the lowering of symmetry from $C_{2 v}$ to $C_{s}$. When a methyl group was substituted for one halogen atom, Ichimura et al. ${ }^{14}$ found that the photodissociation of $o-, m-$, and $p$-chlorotoluene at $193 \mathrm{~nm}$ followed three dissociation channels including direct dissociation and predissociation via ISC or via internal conversion (IC). The methyl substituent enhances the route involving ISC by increasing the rotational levels. The effect of methyl group substitution was also studied for $\mathrm{o}^{-}, \mathrm{m}$-, and $p$-bromotoluene. ${ }^{31,32}$ The substituents affect the coupling strength and the relative energy stability between different states, causing competition among these dissociation channels.

Photodissociation of $o-, m$-, and $p$-bromofluorobenzene have been recently investigated to look into the effects of Fsubstitution and position lying on the benzene ring. ${ }^{20,30}$ By using femtosecond pump-probe spectroscopy with a pumping source at $270 \mathrm{~nm}$, Davidsson and co-workers ${ }^{30}$ found that these molecules have two dissociation channels except for $p$-bromofluorobenzene which has only one, showing similar dissociation pathways as dibromobenzenes. Influence of one $\mathrm{F}$ atom substitution on the dissociation rates is not significant unless a number of $\mathrm{F}$ atoms substitution that accelerates the dissociation processes. The femtosecond laser spectroscopy provides information of dissociation rate constants for the related routes by detecting the parent molecular cations or fragment cations after losing a halogen atom. As an alternative, photofragment translational spectroscopy (PTS) is popularly utilized to understand photodissociation dynamics of aryl halides. ${ }^{11-15,19-21,24,25,29,31,32}$ By means of PTS, Han and co-workers ${ }^{20}$ obtained a quantitative fraction of translational energy partitioned from the available energy and anisotropy parameters in the photolysis of $m$ - and $p$-bromofluorobenzene at $266 \mathrm{~nm}$. They claimed that the $\mathrm{F}$ atom substitution imposes a significant impact on the dissociation mechanism varying parallel transition to perpendicular transition when $\mathrm{F}$ atom moves from meta- to para-position on the ring. 
To the best of our knowledge, velocity imaging detection coupled with resonance-enhanced multiphoton ionization (REMPI) has been much less applied to the investigation of photodissociation of aryl halides except for iodobenzene. ${ }^{33-35} \mathrm{With}$ this technique, the issues of above-threshold effect on dissociative autoionization of superexcited state, ${ }^{33}$ radiation polarization effect on the I-product yield of selective channels, ${ }^{34}$ and alignment dynamics for the spatially oriented iodobenzene were investigated. ${ }^{35}$

By means of the velocity imaging detection, the knowledge of translational energy and angular distributions of the photofragments may be extracted from the obtained fragment images. The branching ratio of spin-orbit ground state and excited halogen may also be determined by selecting particular wavelength in REMPI. By taking advantage of these merits, we aim at the studies of photodissociation of bromofluorobenzenes at $266 \mathrm{~nm}$, in which the F substitution effect has not yet been made certain. The adopted wavelength at $266 \mathrm{~nm}$ lies in a wide excitation range for the $\left(\pi, \pi^{*}\right)$ band. In this work, we observe two dissociation channels for all the molecules. The relative branching ratios for these two routes rely on the F-position. The findings are opposed to the results reported previously. ${ }^{30}$ For the fast dissociation channel, the anisotropy parameters $\beta$ are found to be positive for all the molecules, indicating only one type of parallel transition involvement in the reactions. We do not find any negative value of $\beta$ for para-position as reported by PTS. ${ }^{20}$ For the slow dissociation channel, $\beta$ is very close to zero, suggesting that $\mathrm{Br}$ is isotropically fragmented. The probable dissociation pathways are proposed and compared with those reported by different methods.

\section{Experimental Section}

The velocity imaging apparatus, similar to that used previously, ${ }^{10}$ consisted of a molecular-beam source chamber and a main chamber, both of which were pumped to remain at a low pressure of $\sim 2 \times 10^{-7}$ Torr. Liquid samples of $o^{-}, m-$, and $p$-bromofluorobenzene (99\% purity) were used without further purification. Each was carried by helium gas at $1 \mathrm{~atm}$ through a pulsed valve with $0.6 \mathrm{~mm}$ diameter orifice (General Valve Co.) operating synchronously with the laser pulses at $10 \mathrm{~Hz}$, and expanded into the source chamber. After passing through a $1 \mathrm{~mm}$ diameter skimmer and a collimator, the molecular beam was intersected perpendicularly by a linearly polarized laser beam in a two-stage ion lens region. The skimmer was mounted $30 \mathrm{~mm}$ downstream from the nozzle to divide the source chamber from the main chamber, which comprised an electrostatic lens, a time-of-flight (TOF) drift tube, and a twodimensional position-sensitive detection system. Following the pioneering work of ion velocity map imaging technique by Eppink and Parker, ${ }^{36}$ the set of electrostatic ion lenses was designed to consist of repeller, extractor, and ground electrodes, each with a central hole of 2,16 , and $16 \mathrm{~mm}$ diameter, respectively. The biased voltages were optimized with the aid of simulation using a standard ion optics trajectory programs (Simion 6.0). ${ }^{37}$

A $308 \mathrm{~nm} \mathrm{XeCl} \mathrm{excimer} \mathrm{laser-pumped} \mathrm{dye} \mathrm{laser} \mathrm{(FL3002,}$ Lambda Physik) with pulse duration of 15-20 ns, operating at $10 \mathrm{~Hz}$ was the only radiation source employed. Its output beam was frequency-doubled to emit at about $266 \mathrm{~nm}$ with the energy range of 30-200 $\mu \mathrm{J}$ (AutoTrack III, InRad), followed by linear polarization perpendicular to the flight tube direction, and focused at the skimmed beam with a $200 \mathrm{~mm}$ focal-length lens. The $o-, m-$, and $p$-bromofluorobenzene molecules were then photolyzed and the bromine-atom fragments were successively ionized by using the $(2+1)$ REMPI technique.

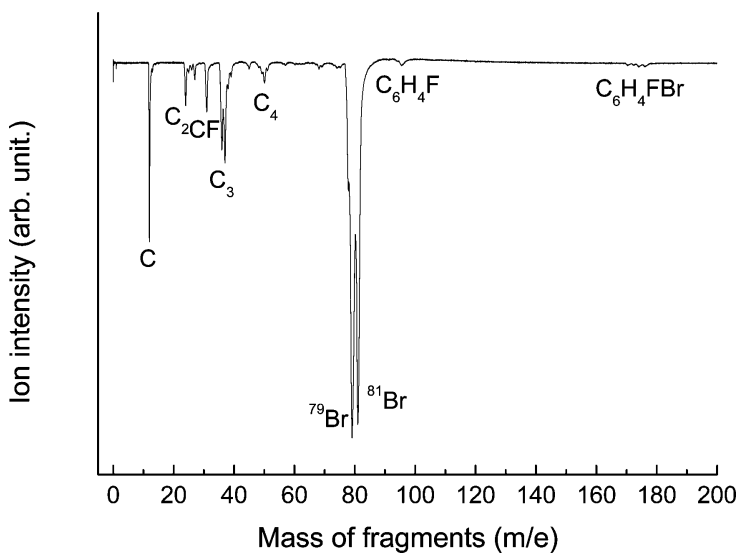

Figure 1. Time-of-flight mass spectrum of photofragments obtained at $266.55 \mathrm{~nm}$ photolysis of 1-bromo-2-fluorobenzene ( $o$-bromofluorobenzene). $\mathrm{Br}^{+}$ion signal is formed by $(2+1)$ REMPI process, and the remaining peaks result from nonresonant ionization.

The resulting ions of $\mathrm{Br}$ or $\mathrm{Br}^{*}$ were extracted and accelerated into a $36 \mathrm{~cm}$ long field-free drift tube along the molecular beam direction. The ion-cloud expansion was driven by both the fragment recoil velocity and the nonhomogeneous electric field formed within the ion lens region, followed by projection onto a two-stage microchannel plate (MCP) and a phosphor screen (FM3040, Galileo). The MCP could be gated within a minimum duration of $250 \mathrm{~ns}$ for mass selection. The ion imaging on the phosphor screen was recorded by a charge coupled device (CCD) camera (200XL4078, Pixelfly). The laser wavelength was scanned back and forth within the range of Doppler broadening to cover all the velocity components of the selected fragments. All the ion signals without gate restriction may be acquired by a photomultiplier tube, instead of the CCD camera, and then transferred to a transient digitizer for display of the TOF mass spectrum. The laser power dependence of the ion intensity was measured directly from the transient digitizer.

\section{Results and Discussion}

A. Time-of-Flight Mass Spectrum. As shown in Figure 1, the TOF mass spectra of $o$-bromofluorobenzene (1-bromo-2fluorobenzene) fragmentation at $\sim 266 \mathrm{~nm}$ are obtained, yielding night peaks at $m / e$ of $12,24,31,36,48,79,81,95$, and 176, corresponding to $\mathrm{C}^{+}, \mathrm{C}_{2}{ }^{+}, \mathrm{CF}^{+}, \mathrm{C}_{3}{ }^{+}, \mathrm{C}_{4}{ }^{+},{ }^{79} \mathrm{Br}^{+},{ }^{81} \mathrm{Br}^{+}, \mathrm{C}_{6} \mathrm{H}_{4} \mathrm{~F}^{+}$, and $\mathrm{C}_{6} \mathrm{H}_{4} \mathrm{FBr}^{+}$, respectively. Among them, the isotope variants ${ }^{79} \mathrm{Br}^{+}$and ${ }^{81} \mathrm{Br}^{+}$are enhanced with $(2+1)$ REMPI via a $5 \mathrm{p}^{4} \mathrm{P}$ ${ }_{3 / 2}^{0} \leftarrow 4 \mathrm{p}^{2} \mathrm{P}_{3 / 2}^{0}$ two-photon transition at $266.55 \mathrm{~nm}$, and the remaining peaks result from the nonresonant processes. Note that the F or HF fragments are not found, because the stronger $\mathrm{C}-\mathrm{F}$ bond cannot be ruptured unless the excitation wavelength is shorter than $196 \mathrm{~nm} \cdot{ }^{26-28}$

When bromofluorobenzenes are photolyzed by using a single laser alone, the bromine atoms may be produced via two probable sources: molecular photodissociation and dissociative ionization, i.e., $\mathrm{C}_{6} \mathrm{H}_{4} \mathrm{FBr}^{+} \rightarrow \mathrm{C}_{6} \mathrm{H}_{4} \mathrm{~F}^{+}+\mathrm{Br}$. The latter process is found in the femtosecond laser experiments, ${ }^{30}$ in which the time-resolved profiles of molecular cations $\mathrm{C}_{6} \mathrm{H}_{4} \mathrm{FBr}^{+}$are probed to determine the lifetimes of the excited molecules before the $\mathrm{Br}$ atom leaves. Nevertheless, in the present nanosecond laser experiment with the pulse energy controlled to be low, the observed $\mathrm{Br}^{+}$in Figure 1 may predominantly come from the photodissociation of the parent molecules in the excited state, followed by ionization via $(2+1)$ REMPI. Because the dissociation lifetimes of the excited state prepared at $266 \mathrm{~nm}$ are in the range of picoseconds, ${ }^{30}$ a great amount of excited 

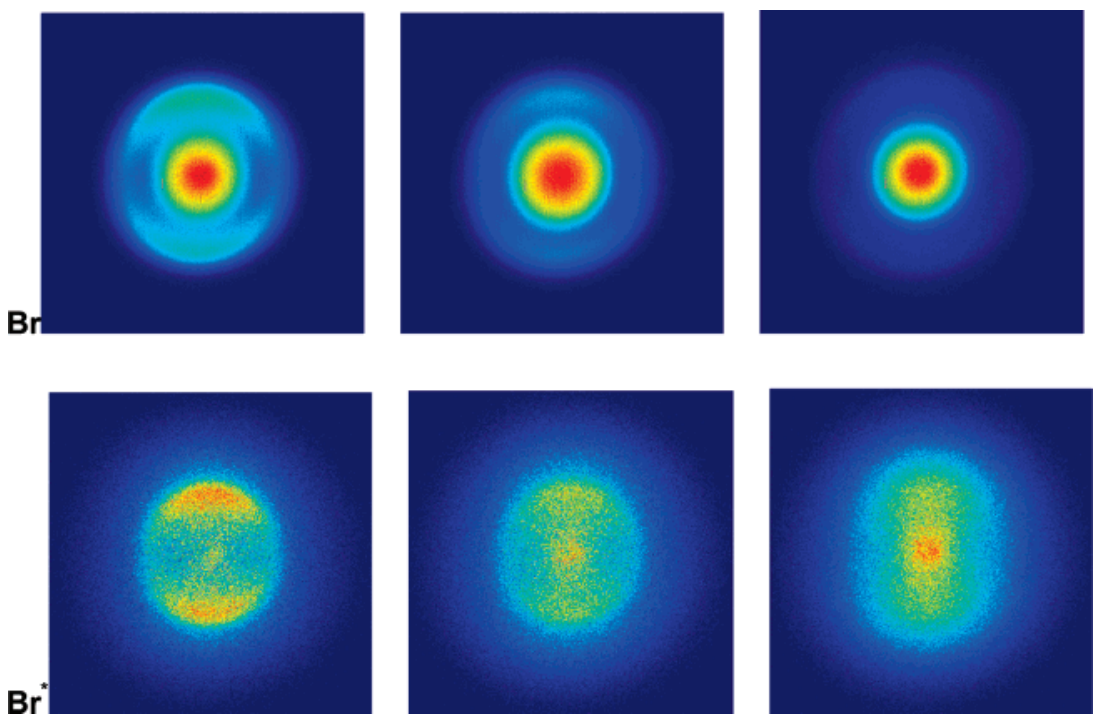

Figure 2. Raw ion images of $\mathrm{Br}^{+}$from photodissociation of $o-, m-$, and $p$-bromofluorobenzene at near $266 \mathrm{~nm}$. The laser is linearly polarized along the vertical direction.
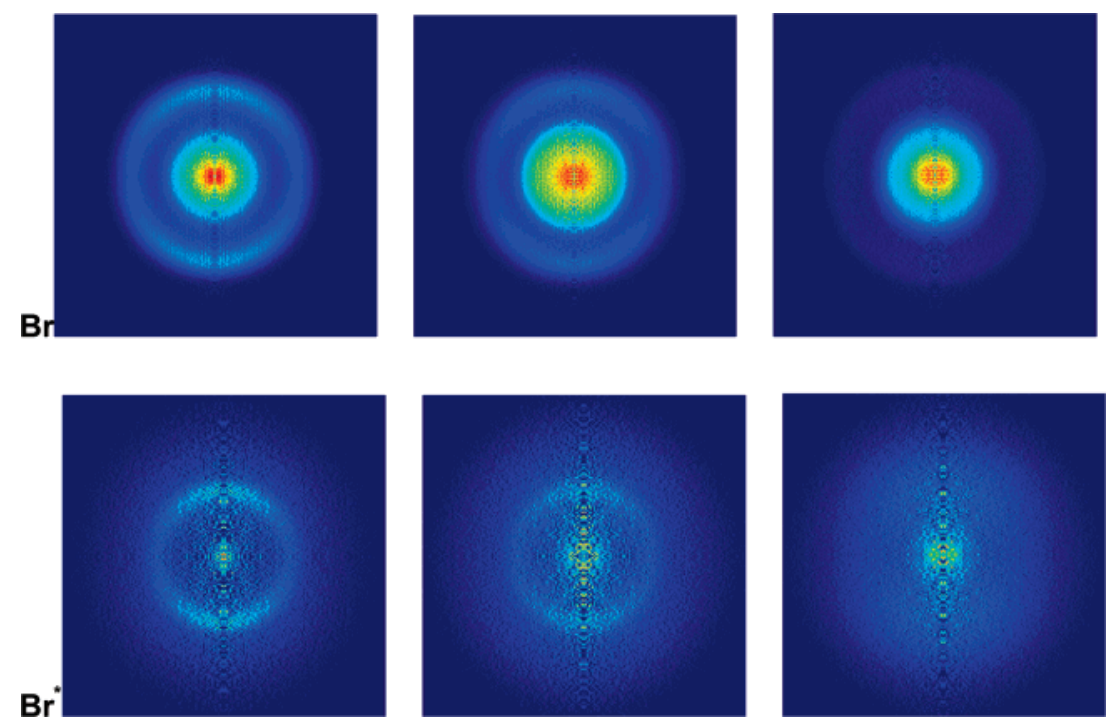

Figure 3. Inverse Abel- transformed ion images of $\mathrm{Br}^{+}$from photodissociation of $o-, m-$, and $p$-bromofluorobenzene at near $266 \mathrm{~nm}$. The laser is linearly polarized along the vertical direction.

molecules have dissociated prior to absorbing an additional photon for ionization. The laser power dependence measurement for the $\mathrm{Br}^{+}$fragment is also against contribution of the dissociative ionization in this work.

B. Translational Energy Distributions and Quantum Yields. Bromofluorobenzenes in this work may be photodissociated to produce the bromine fragments of $\operatorname{Br}\left({ }^{2} \mathrm{P}_{3 / 2}\right)$ and $\mathrm{Br} *\left({ }^{2} \mathrm{P}_{1 / 2}\right)$, followed by ionization with $(2+1)$ REMPI at 266.55 and $266.61 \mathrm{~nm}$, respectively, and then acquired with the velocity imaging detection system. The raw ion images of $\mathrm{Br}$ and $\mathrm{Br}^{*}$ resulting from photodissociation of $\mathrm{o}^{-}, \mathrm{m}$-, and $p$-bromofluorobenzene at near $266 \mathrm{~nm}$ are shown in Figure 2. Each image is accumulated over 40000 laser shots, and the background is removed by subtracting a reference image collected at offresonance wavelength under otherwise the same conditions. Because the obtained $\mathrm{Br}^{*}$ signals are much weaker, their images are accumulated over 100000 laser shots. Each raw image is a two-dimensional projection of the three-dimensional speed and angular distributions with cylindrical symmetry around the polarization axis of the photolyzing laser. According to the method of inverse Abel transformation, the corresponding three- dimensional spatial distributions of the fragments are reconstructed and displayed in Figure 3.

The $\operatorname{Br}\left({ }^{2} \mathrm{P}_{3 / 2}\right)$ ion images associated with $o-, m-$, and $p$ bromofluorobenzene display two distinct components (Figure $3)$. In contrast, the $\mathrm{Br} *\left({ }^{2} \mathrm{P}_{1 / 2}\right)$ images display three components; the outermost ring diffuses broadly, and the middle one shows a clear anisotropic feature.

The speed distribution $P(v)$ can be extracted by integrating the reconstructed three-dimensional speed distribution over all angles at each speed. For bromofluorobenzenes, the center-ofmass translational energy distribution $P\left(E_{\mathrm{T}}\right)$ may be obtained by converting the speed distribution using the following equations:

$$
\begin{gathered}
P\left(E_{\mathrm{T}}\right)=P(v) \frac{\mathrm{d} v}{\mathrm{~d} E_{T}} \\
E_{\mathrm{T}}=\frac{1}{2}\left(m_{\mathrm{Br}}+m_{\mathrm{C}_{6} \mathrm{H}_{4} \mathrm{~F}}\right) \frac{m_{\mathrm{Br}}}{m_{\mathrm{C}_{6} \mathrm{H}_{4} \mathrm{~F}}} v_{\mathrm{Br}}^{2}
\end{gathered}
$$

where $E_{\mathrm{T}}$ is the total translational energy, $m_{\mathrm{X}}$ is the mass of $\mathrm{X}$ ( $\mathrm{X}=\mathrm{Br}$ or $\mathrm{C}_{6} \mathrm{H}_{4} \mathrm{~F}$ ), and $v_{\mathrm{Br}}$ is the velocity of the bromine 
fragment. The available energy $E_{\mathrm{avl}}$ for the dissociation process is evaluated by

$$
E_{\mathrm{avl}}=E_{h v}-D_{0}-E_{\mathrm{el}}+E_{\mathrm{int}}
$$

where $E_{h v}$ is the photon energy, $E_{\mathrm{el}}$ is the electronic energy of the bromine atom, $0 \mathrm{~kJ} \mathrm{~mol}^{-1}$ for $\mathrm{Br}$ and $44 \mathrm{~kJ} \mathrm{~mol}^{-1}$ for $\mathrm{Br}^{*}$, and $E_{\text {int }}$ is the internal energy of the parent molecule. It is expected to be zero because the rotational and vibrational excitations are negligible in a supersonic molecular beam. $D_{0}$ denotes the dissociation energy of the $\mathrm{C}-\mathrm{Br}$ bond, which is evaluated by the PTS method to be $328.8 \mathrm{~kJ} / \mathrm{mol}$ for $m$ bromofluorobenzene and $316.2 \mathrm{~kJ} / \mathrm{mol}$ for $p$-bromofluorobenzene. ${ }^{20}$ A value of $328.8 \mathrm{~kJ} / \mathrm{mol}$ is assumed for $o$-bromofluorobenzene because of its structural similarity with $m$-bromofluorobenzene. The fraction $f_{\mathrm{T}}$ of the translational energy deposition, defined as a ratio of the average translational energy to the available energy, can be determined by

$$
f_{\mathrm{T}}=\frac{\left\langle E_{\mathrm{T}}\right\rangle}{E_{\mathrm{avl}}}
$$

Figure 4 shows the center-of-mass translational energy distributions of $\mathrm{Br}$ and $\mathrm{Br} *$ photofragmentation from $o-, m-$, and $p$-bromofluorobenzene. Each distribution of $\mathrm{Br}$ can be wellfitted by two narrow Gaussian curves, whereas the distribution of $\mathrm{Br}^{*}$ requires one additional broad Gaussian curve. The arrows marked in the figure indicate the maximum kinetic energy available for one-photon dissociation of the parent molecules. Apparently, all the $\mathrm{Br}$ fragments are found to originate from a process of one-photon dissociation, whereas a large fraction of $\mathrm{Br}^{*}$ fragments, especially leading to the broader translational energy component, are produced by two-photon dissociation. The fraction $f_{\mathrm{T}}$ of each translational energy component is evaluated and listed in Table 1.

The obtained bromine ion signal is related to its state population by

$$
\frac{N\left(\mathrm{Br}^{*}\right)}{N(\mathrm{Br})}=k \frac{S\left(\mathrm{Br}^{*}\right)}{S(\mathrm{Br})}
$$

where $N\left(\mathrm{Br}^{*}\right)$ and $N(\mathrm{Br})$ are the numbers of $\mathrm{Br}^{*}$ and $\mathrm{Br}$ fragments; $S\left(\mathrm{Br}^{*}\right)$ and $S(\mathrm{Br})$ are the ion intensities that are measured individually. The factor $k$ is associated with the transition probability ratios in REMPI and the related instrument factors. It can be evaluated by performing a calibration experiment of $\mathrm{Br}_{2}$ photolysis under the same conditions. The $k$ value was thus determined to be 0.595 in the $266 \mathrm{~nm}$ photolysis of $\mathrm{Br}_{2}$. The relative quantum yields of $\Phi\left(\mathrm{Br}^{*}\right)$ and $\Phi(\mathrm{Br})$ can be readily obtained by the following equations,

$$
\begin{gathered}
\Phi\left(\mathrm{Br}^{*}\right)=\frac{N\left(\mathrm{Br}^{*}\right)}{N(\mathrm{Br})+N\left(\mathrm{Br}^{*}\right)} \\
\Phi(\mathrm{Br})=1-\Phi\left(\mathrm{Br}^{*}\right)
\end{gathered}
$$

The results are listed in Table 2. The total $\mathrm{Br}$ fragments dominate over $96 \%$ in the photodissociation of bromofluorobenzenes. $\Phi(\mathrm{Br})_{\text {low }}$ and $\Phi(\mathrm{Br})_{\text {high }}$ are defined as the quantum yield evaluated from the low and high translational energy components as resolved in Figure 4. It is interesting to note that increasing the distance of the fluorine and bromine atoms on the benzene ring leads to increase of $\Phi(\mathrm{Br})_{\text {low }}$ and yet decrease of $\Phi(\mathrm{Br})_{\text {high. }}$ For the $\mathrm{Br}^{*}$ product, the quantum yield is much smaller. If the low and middle translational energy components, which are confined within the maximum kinetic energy (arrow mark), are considered to originate from the one-photon dissociation, their sum amounts to $57 \%, 58 \%$, and $16 \%$ for $o-$, $m$-, and $p$-bromofluorobenzene, respectively, given the values of $\Phi\left(\mathrm{Br}^{*}\right)_{\text {low }}$ and $\Phi(\mathrm{Br} *)_{\text {middle }}$ in Table 2 . When the contribution of the multiphoton effect is neglected, $\Phi(\mathrm{Br})$ may be estimated to be $0.992,0.993$, and 0.995 for the photodissociation of $o-$, $m$-, and $p$-bromofluorobenzene, respectively. $\Phi\left(\mathrm{Br}^{*}\right)_{\text {low }}$ and $\Phi$ $(\mathrm{Br} *)_{\text {middle }}$ obtained at different $\mathrm{Br}$-substituted position exhibit the same trend in magnitude as $\Phi(\mathrm{Br})_{\text {low }}$ and $\Phi(\mathrm{Br})_{\text {high }}$.

C. Angular Distribution. In a photodissociation process, angular distribution $I(\theta)$ of the fragment can be obtained by integrating the reconstructed three-dimensional spatial distribution over a proper range of speed at each angle. It may be characterized by an anisotropy parameter, $\beta$, as expressed by 38,39

$$
I(\theta)=(4 \pi)^{-1}\left[1+\beta P_{2}(\cos \theta)\right]
$$

where $\beta$ is limited between 2 and $-1, P_{2}(\cos \theta)$ is the secondorder Legendre polynomial, and $\theta$ is the angle between the laser polarization direction and the recoil velocity of fragments. The $\beta$ value can be obtained by least-squires fit to the angular distribution. In this manner, the $\beta$ values determined from the $\mathrm{Br}$ and $\mathrm{Br}^{*}$ images at $266 \mathrm{~nm}$ photodissociation are listed in Table 1. It should be noted that information on possible alignment of the $\mathrm{Br}$ fragment may be lost in one-laser experiments. This could slightly affect the measured anisotropy parameter.

For the photodissocation of $o^{-}, m$-, and $p$-bromofluorobenzene, $\beta(\mathrm{Br})$ and $\beta\left(\mathrm{Br}^{*}\right)$ of the lower speed components approximate to zero, indicating that the related dissociation channel is slow enough to produce the isotropic fragments. In contrast, $\beta(\mathrm{Br})$ of the higher speed components and $\beta\left(\mathrm{Br}^{*}\right)$ of the middle speed components are about 0.5 , suggesting that the molecular dissociation results from a parallel transition. The anisotropic feature of the fragments indicates that the dissociation rate is faster than a molecular rotation period.

D. Photodissociation Pathways. By using femtosecond laser spectroscopy with a $270 \mathrm{~nm}$ pump pulse, Davidsson and coworkers $^{30}$ obtained the dissociation lifetimes of (20.8, $\left.5.6 \mathrm{ps}\right)$, (44.1, $15.6 \mathrm{ps}$ ), and $19.5 \mathrm{ps}$ for $o^{-}, \mathrm{m}^{-}$, and $p$-bromofluorobenzene, respectively. $o$ - and $m$-bromofluorobenzene may be photodissociated via two channels, of which the fast channel is blocked in $p$-bromofluorobenzene. The slow dissociation channel for all the molecules is anticipated to involve predissociation via ISC. The dissociation rate relies on location of the curve crossing point and the singlet-triplet coupling strength. With the aid of $a b$ initio one-dimensional potential energy curves along the $\mathrm{C}-\mathrm{Br}$ bond dissociation, ${ }^{30}$ they proposed that the predissociation is via curve crossing between the lowest singlet excited state, $\mathrm{S}_{1}\left(\pi \pi^{*}, \mathrm{~B}_{2}\right)$ (or $\mathrm{S}_{1}\left(\pi \pi^{*}, \mathrm{~A}^{\prime}\right)$ ), and a repulsive triplet state, $\mathrm{T}_{1}\left({ }^{3} \pi \sigma^{*}, \mathrm{~B}_{1}\right)$ (or $\mathrm{T}_{1}\left({ }^{3} \pi \sigma^{*}, \mathrm{~A}^{\prime \prime}\right)$ ). For clear interpretation, a simplified energy diagram for the molecular dissociation is shown in Figure 5, including only the potential energy curves mentioned in this work. The energies at the crossing points relative to the $S_{1}$ minimum state are $0.24,0.21$ and $0.14 \mathrm{eV}$ for $o-, m$-, and $p$-bromofluorobenzene, respectively. Despite insignificant influence on the photodissociation rates by the small energy variation, ${ }^{30}$ the quantum yield $\Phi(\mathrm{Br})_{\text {low }}$, which is associated with this predissociation channel, happens to increase with decreasing the energy level at the crossing point (Table $2)$. In reference to the dissociation lifetime 26 ps of monobromobenzene, ${ }^{22}$ the light $\mathrm{F}$-atom substitution in $\mathrm{o}^{-}, \mathrm{m}$-, and $p$-bromofluorobenzene does not cause significant enhancement in the ISC process. The resultant photodissociation lifetimes 


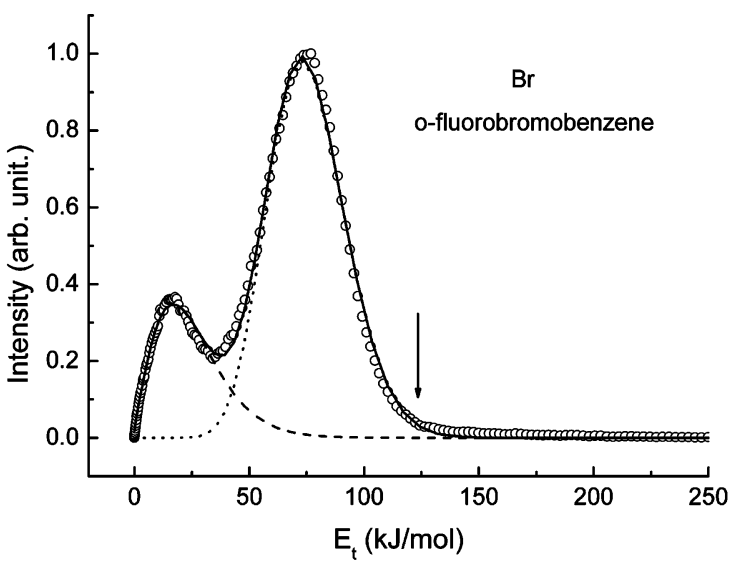

(a)

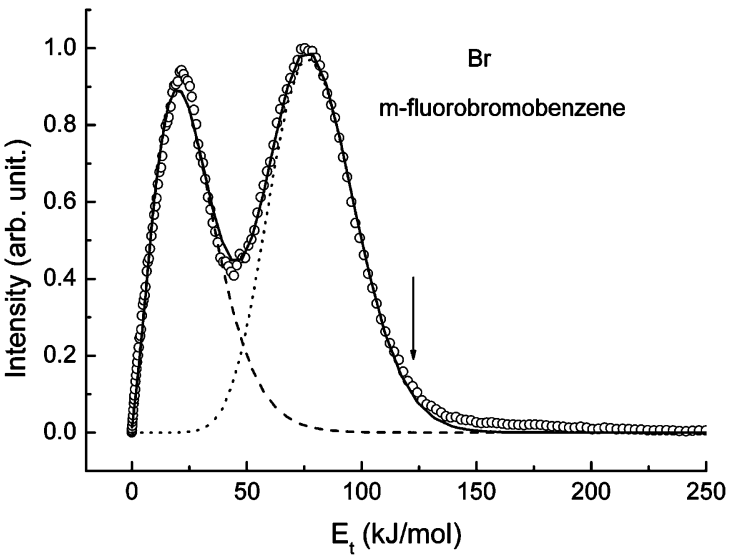

(c)

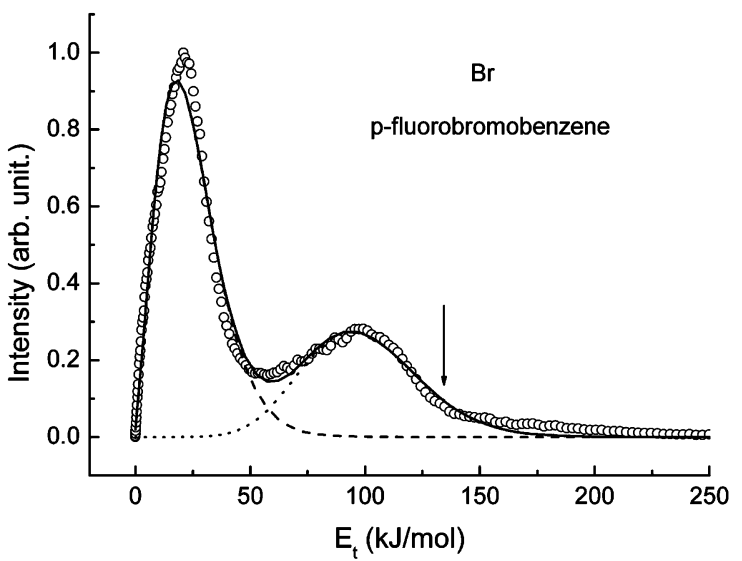

(e)

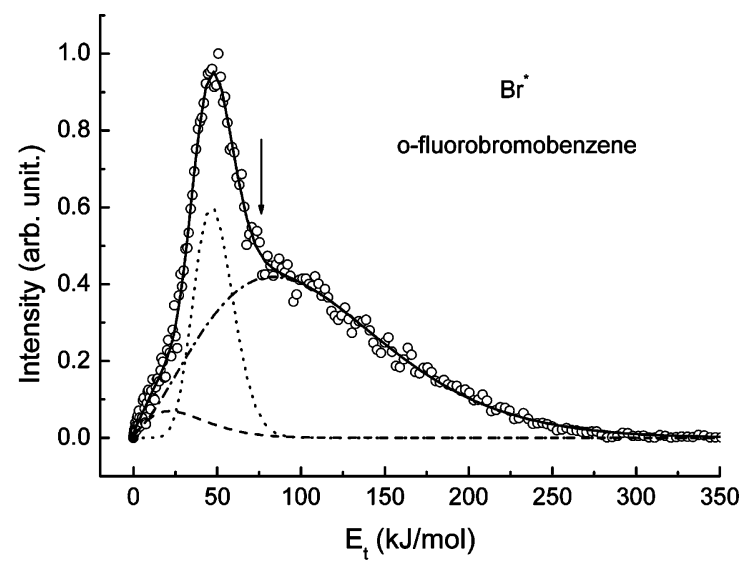

(b)

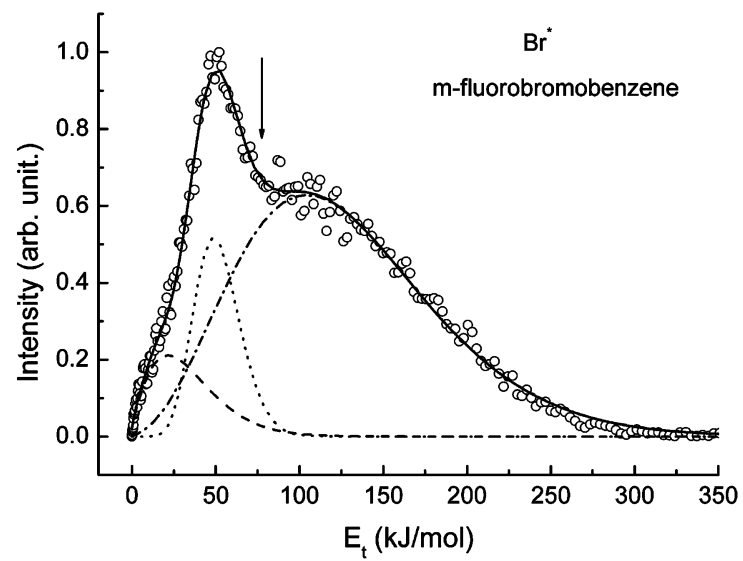

(d)

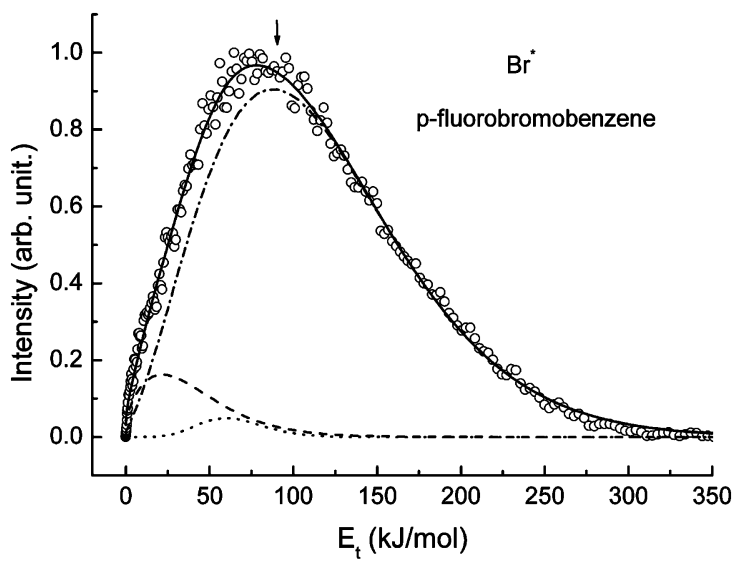

(f)

Figure 4. Total translational energy distributions of the $\mathrm{Br}$ and $\mathrm{Br}$ * fragments produced from photodissociation of $o$-, $m$-, and $p$-bromofluorobenzene at near $266 \mathrm{~nm}$. The arrows indicate the maximum kinetic energy available for one-photon dissociation. Each data point (open circle) corresponds to the relative signal intensity in arbitrary units. In the figures of $\mathrm{Br}$, the low Gaussian components are denoted by the dashed lines (---), and the high Gaussian components are by the dotted lines $(\cdots)$. In Br*, the dashed, the dotted, and the dash-dot lines $(-\cdot-)$ correspond to the low, middle, and high Gaussian components, respectively. In all the figures, the solid lines $(-)$ represent the linear combinations of individual components.

are longer than a molecular rotation period about a few picoseconds, and thus the slowed predissociation rate may lead to production of the isotropic Br fragments. As listed in Table 1 , the $\beta(\mathrm{Br})$ for the lower speed components yields a value close to zero, lending support to the proposed mechanism. In addition, the slow predissociation process allows for more available energy partitioning into the internal states of the fragment. A large fraction of internal energy deposition corresponding to $86 \%, 83 \%$, and $86 \%$ for $o-, m$-, and $p$-bromofluorobenzene, respectively, is obtained.

As for the fast channel for $o$ - and $m$-bromofluorobenzene, Davidsson et al. ${ }^{30}$ proposed the dissociation pathway is via a bound triplet state $\mathrm{T}_{2}\left({ }^{3} \pi \sigma^{*}, \mathrm{~A}^{\prime}\right)$, which has a barrier for dissociation at roughly the same energy as the $\mathrm{S}_{1}-\mathrm{T}_{1}$ ISC point 
TABLE 1: Important Parameters Obtained from Bromine Fragmentation of 2-BrFC $6 \mathrm{H}_{4}, 3-\mathrm{BrFC}_{6} \mathrm{H}_{4}$, and $_{4}-\mathrm{BrFC}_{6} \mathrm{H}_{4}\left(o-, m_{-}\right.$, and $p$-Bromofluorobenzene) at $266 \mathrm{~nm}$

\begin{tabular}{|c|c|c|c|c|c|c|c|}
\hline & state & $\begin{array}{c}h v, \\
\mathrm{~kJ} / \mathrm{mol}\end{array}$ & $\begin{array}{c}E_{a v l} \\
\mathrm{~kJ} / \mathrm{mol}\end{array}$ & $\begin{array}{c}\left\langle E_{\mathrm{t}}\right\rangle, \\
\mathrm{kJ} / \mathrm{mol}\end{array}$ & $f_{\mathrm{T}}$ & $\begin{array}{l}V, \\
\mathrm{~m} / \mathrm{s}\end{array}$ & $\beta$ \\
\hline \multirow[t]{2}{*}{$2-\mathrm{BrFC}_{6} \mathrm{H}_{4}$} & $\mathrm{Br}$ & 449.2 & 120.4 & $\begin{array}{l}17.0 \\
73.1\end{array}$ & $\begin{array}{l}0.14 \\
0.61\end{array}$ & $\begin{array}{l}475.5 \\
986.8\end{array}$ & $\begin{array}{r}-0.01 \pm 0.02 \\
0.55 \pm 0.02\end{array}$ \\
\hline & $\mathrm{Br}^{*}$ & 449.1 & 76.3 & $\begin{array}{l}21.7 \\
46.6 \\
83.5\end{array}$ & $\begin{array}{l}0.28 \\
0.61\end{array}$ & $\begin{array}{r}538.0 \\
788.5 \\
1054.7\end{array}$ & $\begin{array}{l}0.03 \pm 0.02 \\
0.44 \pm 0.04 \\
0.62 \pm 0.1\end{array}$ \\
\hline \multirow[t]{2}{*}{$3-\mathrm{BrFC}_{6} \mathrm{H}_{4}$} & $\mathrm{Br}$ & 449.2 & 120.4 & $\begin{array}{l}19.9 \\
76.9\end{array}$ & $\begin{array}{l}0.17 \\
0.64\end{array}$ & $\begin{array}{r}515.1 \\
1012.2\end{array}$ & $\begin{array}{r}-0.02 \pm 0.02 \\
0.65 \pm 0.03\end{array}$ \\
\hline & $\mathrm{Br}^{*}$ & 449.1 & 76.3 & $\begin{array}{r}21.5 \\
48.8 \\
104.5\end{array}$ & $\begin{array}{l}0.28 \\
0.64\end{array}$ & $\begin{array}{r}535.6 \\
806.1 \\
1180.1\end{array}$ & $\begin{array}{c}-0.03 \pm 0.1 \\
0.49 \pm 0.03 \\
0.5 \pm 0.1\end{array}$ \\
\hline \multirow[t]{2}{*}{ 4- $\mathrm{BrFC}_{6} \mathrm{H}_{4}$} & $\mathrm{Br}$ & 449.2 & 133.0 & $\begin{array}{l}18.0 \\
94.4\end{array}$ & $\begin{array}{l}0.14 \\
0.71\end{array}$ & $\begin{array}{r}489.7 \\
1121.4\end{array}$ & $\begin{array}{r}-0.04 \pm 0.02 \\
0.50 \pm 0.05\end{array}$ \\
\hline & $\mathrm{Br}^{*}$ & 449.1 & 88.9 & $\begin{array}{l}21.9 \\
59.8 \\
87.8\end{array}$ & $\begin{array}{l}0.25 \\
0.67\end{array}$ & $\begin{array}{r}539.8 \\
893.0 \\
1082.0\end{array}$ & $\begin{array}{l}0.01 \pm 0.05 \\
0.5 \pm 0.1 \\
0.65 \pm 0.05\end{array}$ \\
\hline
\end{tabular}

TABLE 2: Relative Quantum Yields of $\mathrm{Br}$ and $\mathrm{Br}^{*}$ for Different Translational Energy Components

\begin{tabular}{cccccccc}
\hline & $\Phi(\mathrm{Br})_{\text {total }}$ & $\Phi(\mathrm{Br})_{\text {low }}$ & $\Phi(\mathrm{Br})_{\text {high }}$ & $\Phi\left(\mathrm{Br}^{*}\right)_{\text {total }}$ & $\Phi\left(\mathrm{Br}^{*}\right)_{\text {low }}$ & $\Phi\left(\mathrm{Br}^{*}\right)_{\text {middle }}$ & $\Phi\left(\mathrm{Br}{ }^{*}\right)_{\text {high }}$ \\
\hline $2-\mathrm{BrFC}_{6} \mathrm{H}_{4}$ & 0.978 & 0.366 & 0.612 & 0.022 & 0.002 & 0.006 & 0.014 \\
$3-\mathrm{BrFC}_{6} \mathrm{H}_{4}$ & 0.981 & 0.554 & 0.427 & 0.019 & 0.003 & 0.004 & 0.012 \\
$4-\mathrm{BrFC}_{6} \mathrm{H}_{4}$ & 0.965 & 0.769 & 0.196 & 0.035 & 0.004 & 0.001
\end{tabular}

described above. The proposed mechanism is referred to Figure 5. $T_{2}\left(A^{\prime}\right)$ with the same symmetry lying energetically close to $\mathrm{S}_{1}\left(\mathrm{~A}^{\prime}\right)$ results in intensity borrowing effect that may enhance the transition probability for $\mathrm{T}_{2}\left(\mathrm{~A}^{\prime}\right) \leftarrow \mathrm{S}_{0}\left(\mathrm{~A}^{\prime}\right)$. They also explained why $p$-bromofluorobenzene in the $C_{2 v}$ symmetry lacks the fast dissociation channel, by attributing to the fact that the $\mathrm{T}_{1}\left(\mathrm{~B}_{2}\right) \leftarrow \mathrm{S}_{0}\left(\mathrm{~A}_{1}\right)$ transition equivalent to $\mathrm{T}_{2}\left(\mathrm{~A}^{\prime}\right) \leftarrow \mathrm{S}_{0}\left(\mathrm{~A}^{\prime}\right)$ is forbidden according to symmetry restriction. In fact, our result differs from that by Davidsson group; the fast dissociation channel in p-bromofluorobenzene is clearly observed (Figure 4). The corresponding quantum yield of $\mathrm{Br}$ with higher translational energy deposition follows a trend along $p-<m$ $<o$-bromofluorobenzene. For $p$-bromofluorobenzene, because $T_{1}\left(B_{2}\right)$ lies energetically close to $S_{1}\left(B_{2}\right)$ and the vibrational modes in multidimensional potential energy surfaces might be involved, the vibronic transition makes probable the fast
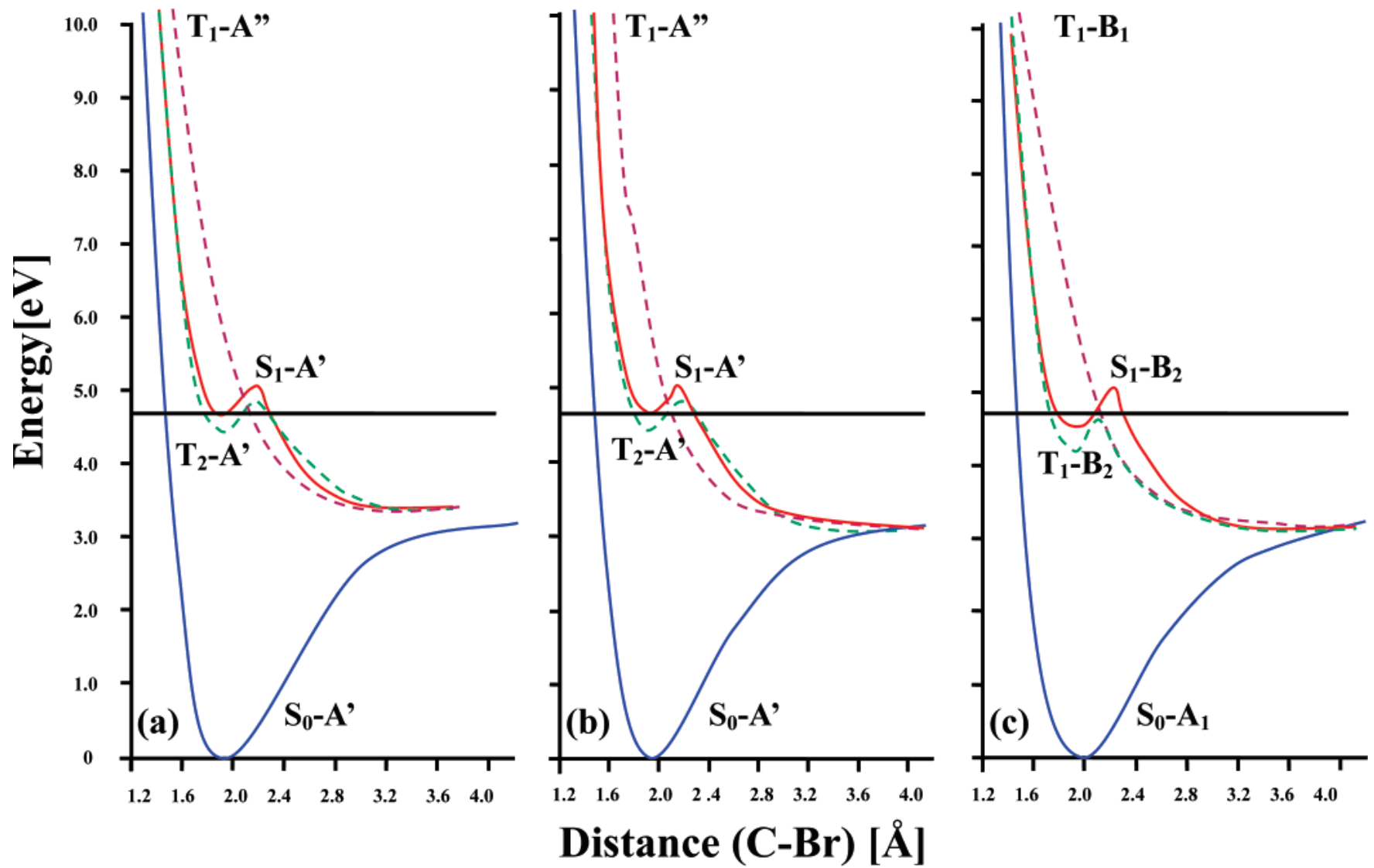

Figure 5. Simplified energy diagrams of one-dimensional potential energy curves along the $\mathrm{C}-\mathrm{Br}$ bond dissociation for (a) $o$-bromofluorobenzene, (b) $m$-bromofluorobenzene, and (c) $p$-bromofluorobenzene. The solid lines represent the singlet states, and the dashed lines denote the triplet states. The excitation energy at $4.66 \mathrm{eV}$ by a laser source is also denoted. The detailed diagrams and calculations are referred to the work by Borg et al. ${ }^{30}$ 

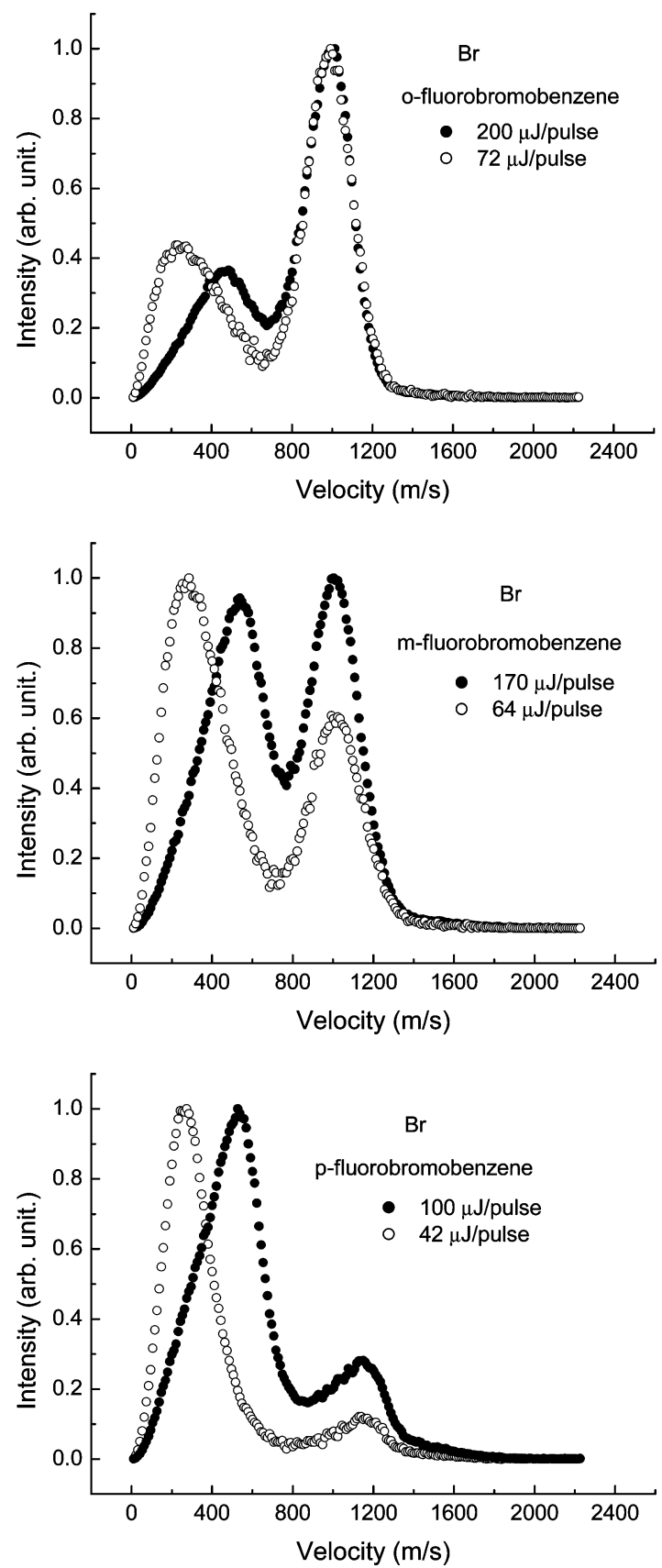

Figure 6. Speed distributions of the $\operatorname{Br}\left({ }^{2} \mathrm{P}_{3 / 2}\right)$ photofragments obtained from $o-, m-$, and $p$-bromofluorobenzenes at different laser energies near $266 \mathrm{~nm}$.

dissociation via the triplet state $\mathrm{T}_{1}\left(\mathrm{~B}_{2}\right)$. According to the $a b$ initio calculations, ${ }^{30}$ the dissociation barrier for this molecule is even smaller than the other two bromofluorobenzenes.

It is worthwhile to note that these two dissociation channels have different response to the laser pulse energy. As shown in Figure 6, when the laser energy is increased to about three times, the peak positions and shapes of the higher translational energy components remain invariant within $9 \%$ uncertainty, except that the intensities increase with respect to the lower energy components. The small position uncertainty is probably caused by the experimental error. In contrast, the profiles become slightly broadened especially for p-bromofluorobenzene and the peak positions shift to higher energy for the lower translational energy distributions. These results are speculated in the following. Increasing laser energy should enhance excitation of population to those vibrational levels with poor Franck-Condon

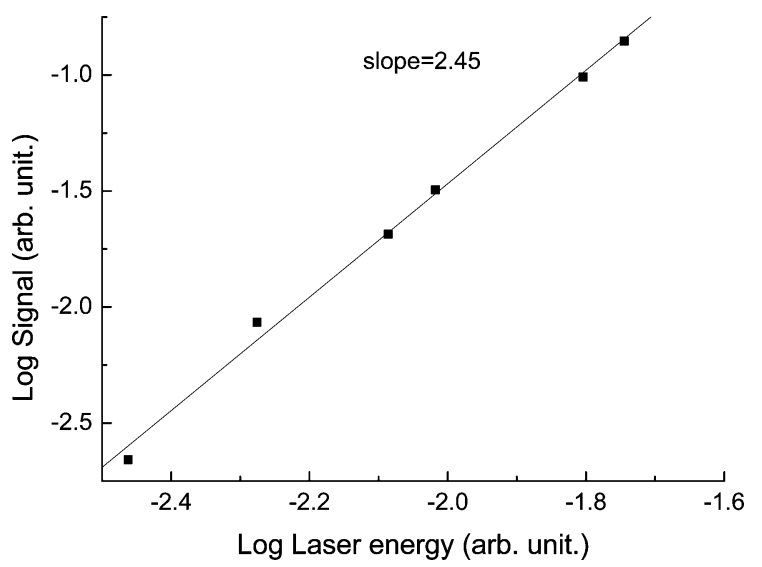

Figure 7. Laser energy dependence of $\mathrm{Br}$ fragmented from $\mathrm{m}$ bromofluorobenzene at $266.55 \mathrm{~nm}$ in a logarithmic scale. The energy range is adjusted to range from 30 to $200 \mu \mathrm{J}$.

factors, including the states either close to or away from the dissociation barrier. The molecules excited in these states have different speeds to surpass the barrier, depending on the energy deficit. The increased amount in the higher vibrational levels gains higher speed to pass through the dissociation barrier at the singlet-triplet crossing point such that the subsequent $\mathrm{Br}$ fragments may carry more translational energy to blue-shift the peak. The translational energy distribution may not remain invariant even after normalization to the laser energy. The increase of laser energy may feasibly cause dissociative ionization, but the extent of increment in this experiment is not large enough to open up this channel significantly. As shown in Figure 7 , the power dependence measurement for $\mathrm{Br}^{+}$fragmented from $m$-bromofluorobenzene at $266.55 \mathrm{~nm}$ yielded a straight line with the slope near 3 , indicating that the ions were obtained via a process of one-photon dissociation, followed by $(2+1)$ REMPI. The final ionization step is partially or completely saturated. The other two bromofluorobenzenes, which lack the data of power dependence measurements, should lead to similar results. If the dissociative ionization process dominates, it will consume at least five photons that are opposed to our power dependence measurement.

In this sense, if the fast dissociation channel is via a bound triplet state $\mathrm{T}_{2}\left(\mathrm{~A}^{\prime}\right)$ or $\mathrm{T}_{1}\left(\mathrm{~B}_{2}\right)$ with a small dissociation barrier as proposed above, ${ }^{30}$ then a similar feature for the profile variation should be observed as in the slow dissociation channel. Such prediction seems to differ from our observation. Accordingly, a more plausible alternative for this dissociation channel is proposed to undergo direct dissociation from a repulsive state $\mathrm{T}_{1}\left(\mathrm{~A}^{\prime \prime}\right)$ or $\mathrm{T}_{1}\left(\mathrm{~B}_{1}\right)$, which lie in energy calculated about $0.5 \mathrm{eV}$ slightly higher than $4.66 \mathrm{eV}$ corresponding to $266 \mathrm{~nm} .^{30}$ Multidimensional potential energy surfaces may have to be involved to explain the probable excitation to this repulsive state. Another possible pathway is by two-photon excitation to a repulsive state, from which the direct dissociation occurs. Then, the maximum $E_{\text {avl }}$ becomes $573.2,573.2$, and $585.5 \mathrm{~kJ} / \mathrm{mol}$ for the photodissociation of $o^{-}, \mathrm{m}^{-}$, and $p$-bromofluorobenzene, respectively. The subsequent fractions of translational energy deposition are substantially reduced to $13-16 \%$, which are difficult to account for a pathway of impulsive dissociation.

Like the case of most alkyl halides, ${ }^{1-10}$ when photodissociation occurs directly along a repulsive state $\left(n, \sigma^{*}\right)$ localized on the $\mathrm{C}-\mathrm{X}$ bond, the obtained translational energy profile of the halogen fragmentation is insensitive to the laser energy variation except for its intensity. Furthermore, the impulsive repulsion causes most available energy partitioning into the translational states. The fraction $f_{\mathrm{T}}$ of translational energy 
deposition for the fast channel obtained in this work is between $61 \%$ and $71 \%$, comparable to those found in the alkyl halides. For instance, $\mathrm{C}_{2} \mathrm{H}_{5} \mathrm{I}$ yields $f_{\mathrm{T}}$ in between 0.62 and 0.75 , when irradiated with a laser source ranging from 258 to $282 \mathrm{~nm} .{ }^{10}$ $p$-Bromofluorobenzene gives rise to a larger $f_{\mathrm{T}}$ of 0.71 , probably because the molecular center-of-mass is along the $C_{2}$ symmetric axis such that the impulsive rupture of the $\mathrm{C}-\mathrm{Br}$ bond may deposit the maximum energy into the translational states.

It is somewhat complicated to comprehend the production channel leading to $\mathrm{Br}^{*}$. We start with an analog in the photodissociation for better interpretation. In A-band photodissociation of $\mathrm{CH}_{3} \mathrm{I}$, the ${ }^{3} \mathrm{Q}_{0}\left(2 \mathrm{~A}_{1}\right)$ state correlates to the $\mathrm{I}^{*}\left({ }^{2} \mathrm{P}_{1 / 2}\right)$ fragment, and the ${ }^{3} \mathrm{Q}_{1}(2 \mathrm{E})$ and ${ }^{1} \mathrm{Q}_{1}(3 \mathrm{E})$ states correlate to $\mathrm{I}\left({ }^{2} \mathrm{P}_{3 / 2}\right){ }^{4,5}$ When ${ }^{3} \mathrm{Q}_{0}\left(2 \mathrm{~A}_{1}\right)$ crosses to ${ }^{1} \mathrm{Q}_{1}(3 \mathrm{E})$, both $\mathrm{I}$ and $\mathrm{I}^{*}$ may be obtained, relying on the state coupling strength. Analogously, the $\mathrm{S}_{1}\left(\pi \pi^{*}, \mathrm{~B}_{2}\right)$ (or $\mathrm{S}_{1}\left(\pi \pi^{*}, \mathrm{~A}^{\prime}\right)$ ) state may correlate to the $\mathrm{Br}^{*}$ product in the photodissociation of bromofluorobenzenes, whereas the $\mathrm{T}_{1}\left({ }^{3} \pi \sigma^{*}, \mathrm{~B}_{1}\right.$ ) (or $\mathrm{T}_{1}\left({ }^{3} \pi \sigma^{*}\right.$, $\left.\mathrm{A}^{\prime \prime}\right)$ ) state correlates to the Br product. As shown in Figure 5, when the $T_{1}$ state is excited, there is a triplet-singlet state coupling in the long-range larger than $\sim 2.8 \AA$, leading to production of both $\mathrm{Br}$ and $\mathrm{Br} *$. According to the Landau-Zener theory, the nonadiabatic surface transition probability should be small when these two surfaces are almost parallel to each other. That might explain why the obtained quantum yield for the $\mathrm{Br}^{*}$ channel is much smaller than that of $\mathrm{Br}$, because the triplet-singlet coupling strength is very weak. For the predissociation pathway, after the $S_{1}-T_{1}$ intersystem crossing at about $2.2 \AA$, the $T_{1}$ state crosses $S_{1}$ again in the exit channel, as in the case of the direct photodissociation. Therefore, as listed in Table 1, the $\mathrm{Br}^{*}$ product results from two pathways similar to the $\mathrm{Br}$ channel, one fast and the other slow with similar fractions of the translational energy deposition. Due to a very small contribution of the perpendicular transition, the obtained values of $\beta$ for the $\mathrm{Br} *$ (middle) channel are slightly smaller than those for $\mathrm{Br}$ (high), except that in $\mathrm{p}$-bromofluorobenzene $\beta$ has the same value but with a large uncertainty. As to the additional broad component of $\mathrm{Br}^{*}$ lying beyond the maximum $E_{\mathrm{avl}}$, the contribution might result from the photodissociation of twophoton absorption of the parent molecules. Nevertheless, we cannot realize why such a multiphoton effect becomes insignificant in the $\mathrm{Br}$ product. Bromofluorobenzenes might happen to cause resonance with some higher dissociative singlet state that leads to the $\mathrm{Br}^{*}$ channel, after absorbing an additional photon at $266 \mathrm{~nm}$.

Han group ${ }^{20}$ have utilized photofragment translational spectroscopy to obtain the $f_{\mathrm{T}}$ values of $46.8 \%$ and $41.7 \%$ as well as $\beta$ of $0.7 \pm 0.1$ and $-0.4 \pm 0.1$ for $m$ - and $p$-bromofluorobenzene, respectively, in the $266 \mathrm{~nm}$ photolysis. The fluorinesubstituted effect does not cause significant influence on the fraction of translational energy deposition, but it changes the $\beta$ orientation. They expected that the parent molecules are excited to a bound $S_{1}$ state which then switches to a repulsive $T_{1}$ state by ISC and finally dissociate. The mechanism corresponds to the slow dissociation pathways proposed by Davidsson et al. ${ }^{30}$ and in this work. While further inspecting the results between Han group and ours, we may find out distinct difference in this channel. We obtain a much smaller $f_{\mathrm{T}}$ of $17 \%$ and $14 \%$ for slow photodissociation of $m$ - and $p$-bromofluorobenzene, and a $\beta$ value almost equal to zero for each molecule. When the fractions of slow and fast translational energy components are averaged with an equal weight assumed (Table 1 ), then $f_{\mathrm{T}}$ of $40.5 \%$ and $42.5 \%$ are obtained for $m$ - and $p$-bromofluorobenzene, close to the results by Han group. Therefore, we speculate their center- of-mass translational energy distributions of the photodissociation at $266 \mathrm{~nm}$ might have mingled two dissociation channels without clear resolution. Their determined $\beta$, which is substantially affected by the center-of-mass translational energy distribution, might not correspond to a particular single dissociation channel. Davidsson and co-workers ${ }^{30}$ obtained the dissociation lifetimes of 44.1 and $19.5 \mathrm{ps}$ for these two molecules, longer than a molecular rotation period, favoring the fragments in an isotropic state in accord with our $\beta$ results.

\section{Conclusion}

$\operatorname{Br}\left({ }^{2} \mathrm{P}_{3 / 2}\right)$ is found to be the primary product in the fragmentation of $o^{-}, \mathrm{m}$-, and $p$-bromofluorobenzene at near $266 \mathrm{~nm}$ by using a photofragment velocity imaging technique combined with a state-selective $(2+1)$ REMPI scheme. The obtained translational energy distributions suggest that the $\mathrm{Br}$ fragments are generated through two dissociation channels. The fast route with a positive anisotropy parameter might be attributed to a direct dissociation, whereas the slow one is initiated from the lowest excited singlet state coupled to a repulsive triplet state. The direct dissociation channel has been recognized in the cases of alkyl halides and monohalobenzenes. This fast channel in $p$-bromofluorobenzene has not been found by femtosecond laser spectroscopy. For the minor product of $\operatorname{Br}\left({ }^{2} \mathrm{P}_{1 / 2}\right)$, the translational energy distributions are fitted by three Gaussian functions, in which the low and middle one result from one-photon dissociation with the dissociating features similar to those observed in $\operatorname{Br}\left({ }^{2} \mathrm{P}_{3 / 2}\right)$, whereas the fast one is from two-photon dissociation. Our kinetic and anisotropic features obtained in photodissociation of $\mathrm{m}$ - and p-bromofluorobenzene differ from those by PTS, with which only one dissociation channel is considered. We believe that difference in the extent of resolution of the translational energy and angular distributions may cause the discrepancy. In the velocity imaging technique, these two parameters may be independently extracted from the photofragment ion images.

Acknowledgment. Y.T. thanks the financial support of Chinese Foundation to visit with Professor Lin's group. This work is supported by National Science Council of Taiwan, Republic of China under contract no. NSC 96-2113-M-002027.

\section{References and Notes}

(1) Johnson, B. R.; Kittrell, C.; Kelly, P. B.; Kinsey, J. L. J. Phys. Chem. 1996, 100, 7743

(2) Amatatsu, Y.; Yabushita, S.; Morokuma, K. J. Chem. Phys. 1996, 104, 9783.

(3) Samartzis, P. C.; Bakker, B. L.; Parker, D. H.; Kitsopoulos, T. N. J. Phys. Chem. A 1999, 103, 6106.

(4) Eppink, A. T. J. B.; Parker, D. H. J. Chem. Phys. 1999, 110, 832.

(5) Xu, H.; Guo, Y.; Liu, S.; Ma, X.; Dai, D.; Sha, G. J. Chem. Phys. 2002, 117, 5722 .

(6) Wu, G.; Jiang, B.; Ran, Q.; Zhang, J.; Harich, S. A.; Yang, X. J. Chem. Phys. 2004, 120, 2193.

(7) Philips, D. L.; Lawrence, B. A.; Valentini, J. J. J. Phys. Chem. 1991, 95, 9085

(8) Rattigan, O. V.; Shallcross, D. E.; Cox, R. A. J. Chem. Soc., Faraday Trans. 1997, 93, 2839.

(9) Tang, Y.; Ji, L.; Zhu, R.; Wei, Z.; Zhang, B. Chem. Phys. Chem. 2005, 6, 2137

(10) Tang, Y.; Lee, W. B.; Hu, Z.; Zhang, B.; Lin, K. C. J. Chem. Phys. 2007, 126, 064302.

(11) Dzvonik, M.; Yang, S. C.; Bersohn, R. J. Chem. Phys. 1974, 61, 4408 .

(12) Freedman, A.; Yang, S. C.; Kawasaki, M.; Bersohn, R. J. Chem. Phys. 1980, 72, 1028

(13) Ichimura, T.; Mori, Y.; Shinohara, H.; Nishi, N. Chem. Phys. Lett. 1986, $125,263$. 
(14) Ichimura, T.; Mori, Y.; Shinohara, H.; Nishi, N. J. Chem. Phys. 1997, 107, 835.

(15) Hwang, H. J.; El-Sayed, M. A. J. Chem. Phys. 1992, 96, 856. 35 .

(17) Kadi, M.; Davidsson, J. Chem. Phys. Lett. 2003, 378, 172.

(18) Liu, Y. J.; Persson, P.; Karlsson, H. O.; Lunell, S.; Kadi, M.; Karlsson, D.; Davidsson, J. J. Chem. Phys. 2004, 120, 6502.

(19) Zhang, H.; Zhu, R. S.; Wang, G. J.; Han, K. L.; He, G. Z.; Lou, N. Q. J. Chem. Phys. 1999, 110, 2922.

(20) Gu, X. B.; Wang, G. J.; Huang, J. H.; Han, K. L.; He, G. Z.; Lou, N. Q. J. Phys. Chem. A 2001, 105, 354.

(21) Ichimura, T.; Mori, Y.; Shinohara, H.; Nishi, N. Chem. Phys. 1994, $189,117$.

(22) Kadi, M.; Davidsson, J.; Tarnovsky, A. N.; Rasmusson, M.; Akesson, E. Chem. Phys. Lett. 2001, 350, 93.

(23) Pence, W. H.; Baughcum, S. L.; Leone, S. R. J. Phys. Chem. 1985, 85,3844 .

(24) Freitas, J. E.; Hwang, H. J.; El-Sayed, M. A. J. Phys. Chem. 1995 $99,7395$.

(25) Griffiths, J. A.; Junk, K.; El-Sayed, M. A. J. Phys. Chem. 1996, 100,7989

(26) Liu, Y. J.; Persson, P.; Lunell, S. J. Phys. Chem. A 2004, 108, 2339.
(27) Huang, C. L.; Jiang, J. C.; Mebel, A. M.; Lee, Y. T.; Ni, C. K. J. Am. Chem. Soc. 2003, 125, 9814.

(28) Lee, S. H.; Wu, C. Y.; Yang, S. K.; Lee, Y. P. J. Chem. Phys. 2006, 125, 144301 .

(29) Zhu, R. S.; Zhang, H.; Wang, G. J.; Gu, X. B.; Han, K. L.; He, G. Z.; Lou, N. Q. Chem. Phys. 1999, 248, 285.

(30) Borg, O. A.; Liu, Y. J.; Persson, P.; Lunell, S.; Karlsson, D.; Kadi, M.; Davidsson, J. J. Phys. Chem. A 2006, 110, 7045.

(31) Zhang, H.; Zhu, R. S.; Wang, G. J.; Han, K. L.; He, G. Z.; Lou, N. Q. Chem. Phys. Lett. 1999, 300, 483.

(32) Gu, X. B.; Wang, G. J.; Huang, J. H.; Han, K. L.; He, G. Z.; Lou, N. Q. Chem. Phys. 2003, 287, 285.

(33) Unny, S.; Du, Y.; Zhu, L.; Truhins, K.; Gordan, R. J.; Sugita, A. Kawasaki, M.; Matsumi, Y.; Delmdahl, R.; Parker, D. H.; Berces, A. J. Phys. Chem. A 2001, 105, 2270.

(34) Poulsen, M. D.; Skovsen, E.; Stapelfeldt, H. J. Chem. Phys. 2002 117, 2097.

(35) Peronne, E.; Poulsen, M. D.; Stapelfeldt, H.; Bisgaard, C. Z.; Hamilton, E.; Seideman, T. Phys. Rev. A 2004, 70, 063410.

(36) Eppink, A. T. J. B.; Parker, D. H. Rev. Sci. Instrum. 1997, 68, 3477.

(37) Dahl, D. A.; Delmore, J. E.; Appelhans, A. D. Rev. Sci. Instrum. 1990, 61, 607

(38) Zare, R. N.; Herschbach, D. R. Proc. IEEE. 1963, 51, 173.

(39) Zare, R. N. Mol. Photochem. 1972, 4, 1. 